
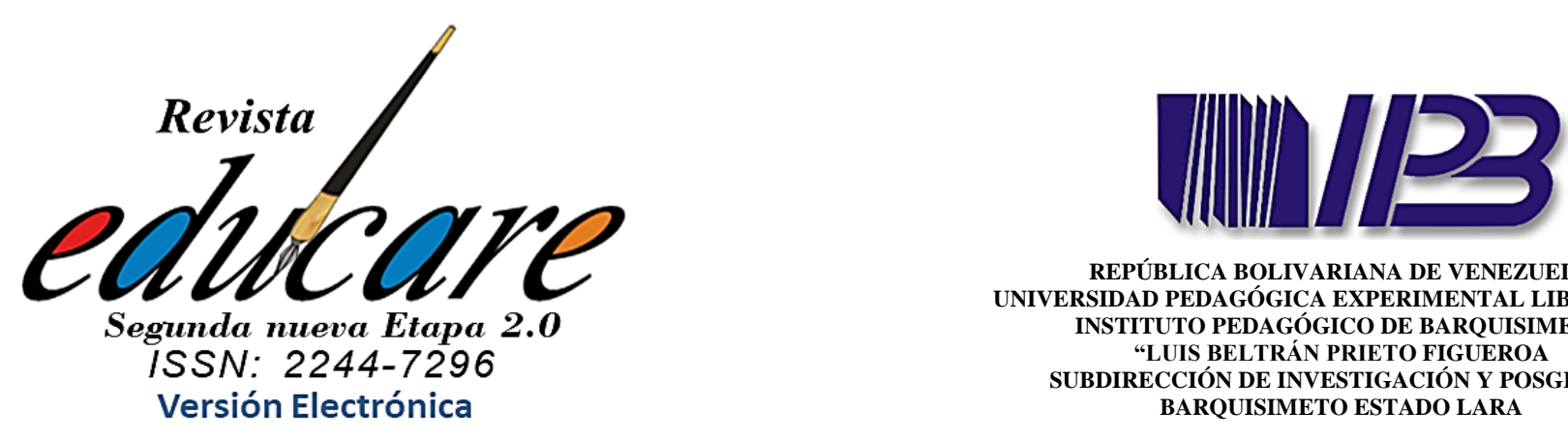

REPÚBLICA BOLIVARIANA DE VENEZUELA UNIVERSIDAD PEDAGÓGICA EXPERIMENTAL LIBERTADOR INSTITUTO PEDAGÓGICO DE BARQUISIMETO "LUIS BELTRÁN PRIETO FIGUEROA SUBDIRECCIÓN DE INVESTIGACIÓN Y POSGRADO BARQUISIMETO ESTADO LARA

Volumen 24 № 1 Enero-Abril 2020

$(51-74)$

Marisol Sellet Zurita Aguilera*

ORCID: https://orcid.org/0000-0002-2347-2575.

UNIVERSIDAD NACIONAL

EXPERIMENTAL DE LA FUERZA

ARMADA (UNEFA)

VENEZUELA

* Licenciada en Educación mención Planificación Educativa de la Universidad Central de Venezuela (UCV);

Especialista en Evaluación Educacional (UPEL); Magíster Scientiarum en Ciencias de la Educación, mención Psicología de los Procesos en el Aula (UNESR), Doctora en Innovaciones Educativas. Profesora a Dedicación Exclusiva de la UNEFA. Correo electrónico margasam4@gmail.com

\section{EL APRENDIZAJE COOPERATIVO Y EL DESARROLLO DE LAS HABILIDADES COGNITIVAS}

\author{
COOPERATIVE LEARNING AND THE \\ DEVELOPMENT OF COGNITIVE SKILLS
}

Recibido:

27-10-2018

Aceptado:

30-11-2019 

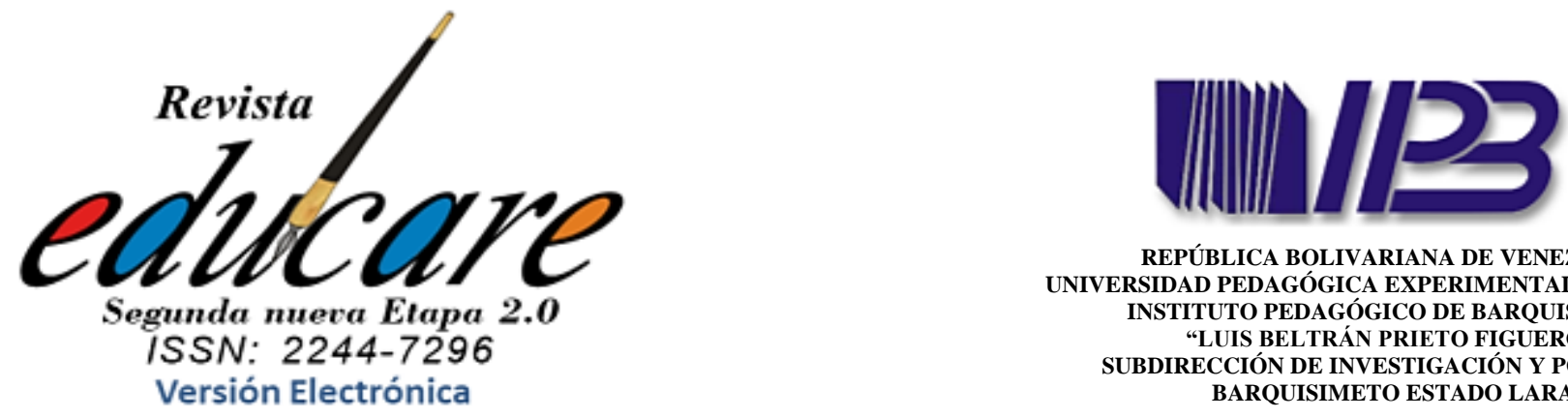

REPÚBLICA BOLIVARIANA DE VENEZUELA UNIVERSIDAD PEDAGÓGICA EXPERIMENTAL LIBERTADOR INSTITUTO PEDAGÓGICO DE BARQUISIMETO "LUIS BELTRÁN PRIETO FIGUEROA

SUBDIRECCIÓN DE INVESTIGACIÓN Y POSGRADO BARQUISIMETO ESTADO LARA

\section{EL APRENDIZAJE COOPERATIVO Y EL DESARROLLO DE LAS HABILIDADES COGNITIVAS}

\author{
COOPERATIVE \\ LEARNING AND THE \\ DEVELOPMENT OF \\ COGNITIVE SKILLS
}

\section{Resumen}

El objetivo de la investigación es comprender la relación entre el aprendizaje cooperativo y el desarrollo de las habilidades cognitivas en estudiantes de Educación Básica. La metodología de investigación utilizada fue la cualitativa, a través de la observación y entrevistas como técnicas para la recolección de la información; la experiencia se desarrolló en el área de Educación Artística durante los tres lapsos del año escolar en una sección de estudiantes. Dentro de los hallazgos encontrados se señala que el aprendizaje cooperativo facilita que los estudiantes manifiesten lo que aprenden con mayor soltura, mediante actividades comprensivas de explicación, descripción, ejemplificación y contextualización. Una de las conclusiones de interés es que la interacción en equipos cooperativos contribuye a desarrollar habilidades cognitivas. En la medida que los estudiantes socializan adquieren, elaboran $y$ transfieren el conocimiento y sus aprendizajes.

Descriptores: Aprendizaje cooperativo, Habilidades cognitivas, Interacción

\begin{abstract}
The objective of the research is to understand the relationship between cooperative learning and the development of cognitive skills in Basic Education students. The methods were applied in a student section during a school year. The research methodology used was the qualitative; through observation and interviews as techniques for gathering information; the experience was developed in the area of Arts Education during the three lapses of the school year. Among the findings found it is noted that cooperative learning makes it easier for students to express what they learn most freely, through comprehensive activities of explanation, description, exemplification and contextualization. One of the interesting conclusions is that interaction in cooperative teams contributes to the development of cognitive skills. As students socialize, they acquire, elaborate and transfer knowledge and their learning.
\end{abstract}

Keywords: Cooperative learning. Cognitive skills, Interaction 


\section{INTRODUCCIÓN}

La construcción del conocimiento en las aulas tiene su origen en la construcción social, ya que "el aprendizaje supone un carácter social determinado y un proceso por el cual los estudiantes inician su desarrollo intelectual a través de aquellos que les rodean" (Morales, García, Torre y Lebrija, 2018, p.47), además está regido por "la articulación y negociación entre las intervenciones de los docentes y la participación pasiva y activa de los estudiantes" (Candela, 1995, p.173). La participación del alumnado en todas las actividades escolares favorece el aprendizaje significativo, tanto de los contenidos adquiridos durante las interacciones, como de las habilidades cognitivas y sociales, contribuyendo al desarrollo del pensamiento crítico y creativo en la resolución de problemas, como sugiere Flores (2015).

Bajo este supuesto, el logro del aprendizaje requiere de la participación activa de la persona que aprende. Una participación limitada del estudiantado en sus procesos de aprendizajes no le ayuda en el progreso de sus habilidades; por consiguiente, el aprendizaje cooperativo hace que los estudiantes sean "agentes activos en el proceso de aprendizaje en lugar de receptores pasivos del producto de cualquier conocimiento cierto" (Loor, Palma, Saltos y Bolívar, 2018, p.435). De manera, que la relación entre el estudiantado y el conocimiento está mediada por el docente y por sus pares, mediante el proceso de interacción donde ponen en común sus experiencias influenciadas por el contexto cultural en el cual se desenvuelven.

En este sentido, el trabajo en equipo mediante el aprendizaje cooperativo como mediador, mejora las relaciones entre los estudiantes e incrementa sus habilidades cognitivas; es una herramienta que beneficia el aprendizaje. Su aplicación incide de manera positiva en las relaciones entre los estudiantes (Pujolás, 2011), y contribuye a enfrentar la carencia de habilidades cognitivas que representan un obstáculo para los estudiantes de cualquier nivel educativo (Papalia, Wendkos y Duskin, 2010).

En los sistemas educativos actuales se descubren innumerables problemas que afectan el desarrollo de las habilidades cognitivas, siendo los más resaltantes los siguientes: el uso de textos muy extensos, dificultades para comprender, actividades académicas sustentadas en estrategias memorísticas, estrategias centradas en el docente y en donde su opinión prevalece 
ante la de los estudiantes, trabajos en equipos sin controles que promueven la falta de solidaridad y comprensión entre sus miembros, exposiciones memorísticas sin reflexión, calificaciones deficientes y poca comprensión de los textos escritos, manifestándose en frustraciones e inadecuada formación del estudiantado (Ramos, Herrera y Ramírez, 2010; Ríos 2014; Capilla 2016).

Aunado a esto, en la Educación Básica y Diversificada se presenta un problema relacionado entre el tiempo y el número de objetivos de las asignaturas. Muchas veces a los profesores se les dificulta cubrir la totalidad del programa, sea por su extensión, complejidad, niveles cognoscitivos de los estudiantes, entre otros, dificultándole el logro de las pautas planificadas en un principio (Martinic, 2015). En este sentido, es pertinente abordar métodos que respondan a situaciones como la antes descrita, siendo necesario considerar el uso de habilidades cognitivas mediante metodologías interactivas que faciliten el progreso social del conocimiento y del lenguaje. Por ello, se formula la siguiente interrogante de investigación: ¿Cómo se relaciona el aprendizaje cooperativo con el desarrollo de las habilidades cognitivas en estudiantes de Educación Básica?

En relación con lo anterior se presentan los siguientes objetivos de investigación: describir el proceso de aplicación del aprendizaje cooperativo en el desarrollo de habilidades cognitivas en estudiantes de Educación Básica y comprender la relación entre el aprendizaje cooperativo y el desarrollo de las habilidades cognitivas.Para el logro de los mismos se llevó a cabo un proceso metodológico cualitativo, realizándose observaciones y entrevistas que luego fueron categorizadas, analizadas y debidamente interpretadas.

\section{APORTES TEÓRICOS}

\section{Aprendizaje Cooperativo: Características y Principios}

El aprendizaje cooperativo es un sistema didáctico que potencializa las capacidades intelectuales y las habilidades sociales (Slavin, 1987; Ovejero, 1990). Son métodos interactivos que favorecen el desarrollo cognitivo - afectivo. Para Aranguren (2014) se caracterizan por la interacción entre estudiantes que trabajan juntos para aprender. Su práctica supone, en el estudiantado, la descentración cognitiva modificadora de las estructuras intelectuales dando lugar al progreso cognitivo. Las metas del aprendizaje cooperativo son estrictamente grupales, su éxito depende de cada uno de sus miembros; por lo que el intercambio de ideas, posturas e 
información, propician la interacción generando la confrontación de puntos de vistas en torno a una misma tarea.

El aprendizaje cooperativo se sostiene en diversos principios fundamentales (Slavin, 1999; Johnson, y Johnson, 1999; Azorín 2018), demostrables en el ejercicio práctico de los métodos: la interdependencia positiva, la interacción cara a cara, el desarrollo de habilidades cognitivas y sociales y la valoración personal. La interdependencia positiva representa el vínculo estudiante-grupo, por lo que el éxito grupal depende de esta interacción; la interacción cara a cara consiste en el avance del trabajo entre estudiantes talentosos y otros menos aventajados; el desarrollo de las habilidades cognitivas y sociales son necesarias para el logro del aprendizaje, y todos los miembros de los equipos deben ser capaces de demostrarlo y por último, la valoración personal requiere una evaluación que busca asegurar que cada estudiante sea estimado por sus esfuerzos y contribuciones.

El grado de igualdad en los roles a cumplir por cada miembro del grupo, conecta y profundiza en sus competencias comunicativas (García, 2018). La mutualidad en los grupos promueve la planificación y discusión en conjunto, favorece el intercambio de roles y delimita la división del trabajo. Una práctica por períodos prolongados ayuda a desarrollar hábitos intelectuales y sociales para la construcción del conocimiento.

Durante la aplicación del aprendizaje cooperativo deben verificarse, de manera demostrativa y observable, el ejercicio de los principios básicos que lo sustentan. Siendo necesario el rol de mediador y facilitador del docente, y que los estudiantes se conviertan en facilitadores de sus aprendizajes. Es importante la heterogeneidad de los equipos, la interdependencia positiva, la autonomía grupal y valoración del liderazgo temporal, así como la adquisición de habilidades, tanto cognitiva como sociales, que se desarrollan, de manera integrada, en la medida que se dan los procesos internos en los equipos.

\section{Las Habilidades Cognitivas}

Un niño, atraviesa cuatro estadios en su proceso de desarrollo: el sensomotor, el preoperatorio, el de las operaciones concretas y el pensamiento formal (Piaget e Inhelder, 2015), evidenciadas por una serie de características, conductas, destrezas y habilidades que distinguen el alcance de su madurez intelectual. En todo esto no existe un esquema de desarrollo intelectual rígido que supone creer que todos los seres experimentan el desarrollo de la misma manera, más bien es una construcción progresiva dada por las innovaciones que 
surgen en función de lo precedido; lo preestablecido es una construcción de los modelos de pensamiento del adulto, que el niño solo comprende cuando lo reconstruye convirtiéndose en una operación ininterrumpida, es un desarrollo con dimensiones: ontogenética y social.

Las habilidades intelectuales son adquiridas y modificadas mediante procesos de adaptación constituidos por los sub-procesos de asimilación y acomodación. La persona es capaz de organizar y reorientar las habilidades con el objeto de responder al mundo que le rodea. Son operaciones asociadas a la realización de determinadas acciones que puede ejecutar el sujeto, es decir, se procesa la información, luego se comprende, analiza, argumenta y se producen nuevos enfoques, por lo que al estar los sujetos trabajando juntos y confrontando puntos de vista diferentes, crean conflictos que generan nuevos aprendizaje (Mugny, 1988; Samaniego, 1995; Azorín, 2018). La asimilación de habilidades está acompañado de procesos cognoscitivos que exige la atención voluntaria y consciente; la asimilación real del sistema de acciones que la conforman, así como del conocimiento al cual está asociada, además, su formación y desarrollo, supone de los estudiantes la comprensión del significado y el valor de estas habilidades para el propio proceso del conocer.

Las habilidades cognitivas son un conjunto de operaciones mentales que integran la información adquirida mediante los sentidos en una estructura de conocimientos significativos, de modo que el estudiante no está limitado al proceso de adquisición sino que es capaz de construir el conocimiento haciendo uso de la experiencia previa para la comprensión y precisión del nuevo aprendizaje. Romero y Tapia (2014) las definen como destrezas y procesos de la mente útiles para la realización de tareas porque responden a los procesos de adquisición y de recuperación, por lo que pueden ser utilizadas para ser desarrolladas con cualquier contenido en el aula.

El desarrollo de las destrezas cognitivas supone transformaciones y relaciones que son útiles al estudiante para su interacción con el ambiente; por consiguiente, las habilidades adquiridas por el estudiante le funcionan para su interacción el contexto mediante símbolos, es por ello que aprenden "cómo transformar los símbolos impresos en una página en palabras reconocibles, cómo convertir fracciones en decimales, cómo establecer concordancia entre verbos y pronombres en una oración, cómo transformar una expresión inglesa a una expresión en español" (Chadwick y Rivera, 1991, p.84). 
En concordancia con lo anterior, las habilidades intelectuales son "las capacidades que hacen posible el uso de símbolos" (Gagné, 1987, p.50), es decir, facilitan el desarrollo de la abstracción, dado que ésta es la capacidad sostenida en el uso de las representaciones simbólicas que se oponen a lo concreto. Mediante la abstracción se capta lo esencial y las propiedades del objeto pensado, manteniendo la diversidad de aspectos en la mente, asociándolas y procesándolas ante una respuesta; por tanto, en los infantes el pensamiento abstracto se organiza a medida que se especializan las percepciones (Pérez, 2016).

Ahora bien, siguiendo la lógica de Gagné las habilidades intelectuales se adquieren en un orden que va desde la discriminación, hasta reglas de orden superior, pasando por la conceptualización y el desarrollo de estas reglas. Este mismo principio se descubre en el programa de desarrollo del pensamiento (De Sánchez, 1991), que parte del supuesto de que pensar es una habilidad desarrollada a partir de la percepción, o sea, de la manera de ver el mundo. Esto supone el dominio de operaciones que involucran el recuerdo, la comprensión, la resolución de problemas, el seguimiento de reglas e instrucciones, la definición de conceptos y el reconocimiento de estímulos (Vergel, Duarte y Martínez, 2016). Con el desarrollo de habilidades intelectuales se flexibiliza el pensamiento, se regula la impulsividad, reestructuración y organización de los patrones de pensamiento, facilita la toma de decisiones, argumentar, las relaciones interpersonales y las habilidades para manejar la información.

Las habilidades cognitivas son estrategias adquiridas durante largos períodos de tiempo, funcionan para atender, aprender, pensar y resolver problemas; abarcan no solo el sistema de adquisición, sino también el almacenamiento de la información, su transferencia y resolución de problemas, porque permiten interpretar las situaciones cotidianas que la persona enfrenta a diario (Muñoz, 2017). En este sentido, las habilidades cognitivas se clasifican en dos: habilidades cognitivas generales y habilidades cognitivas relacionadas con un dominio de conocimiento:

Las primeras tienen un carácter más general, ya que se supone actúan de forma similar a través de los diferentes dominios de conocimientos; las segundas se desarrolla en estrecha relación con la adquisición de contenidos específicos o han sido estudiadas dentro de un dominio particular (Gilar, 2003, pp.8-9).

De acuerdo al mismo autor, las habilidades cognitivas generales se adquieren mediante tres fases: inicial, intermedia y final. Siendo la inicial aquella dada a la adquisición del conocimiento; la intermedia se refiere al procesamiento de la información y la final es la 
recuperación, generalización, transferencia y sistematización de la información. Estas habilidades deben ser demostrables, por lo que una forma de hacerlo es mediante las llamadas actividades de comprensión: la explicación, la ejemplificación, la aplicación, la justificación, la comparación y el contraste, la contextualización y la generalización (Perkins, 1999; Hernández y Aranguren, 2016), en este sentido, la comprensión es una actividad mental creativa que va más allá de la información suministrada, es considerada un estado de capacitación de la mente.

\section{METODOLOGÍA}

\section{Diseño de Investigación}

El diseño de investigación es de campo y de tipo cualitativo. Se realizó un estudio de las interacciones dadas en los grupos de aprendizaje cooperativo conformados en un aula de clases durante el desarrollo de la cátedra de Educación Artística. Fue necesario acompañar a la docente y al estudiantado durante un año escolar, dividido en tres períodos: durante el primero se realizaron observaciones de las actividades planificadas por el docente, en el segundo se aplicaron las actividades de aprendizaje cooperativo en cada clase y el tercero fue para legitimar la información obtenida.

La institución donde se llevó a cabo la experiencia está ubicada al este de la ciudad de Caracas y atiende a una población de estudiantes provenientes de familias constituidas por profesionales y comerciantes con negocios independientes. La escuela conduce estudios desde el 7mo grado de Educación Básica al 2do año de Ciclo Diversificado. Para esta investigación se consideraron 25 estudiantes cursantes del 8vo grado de Educación Básica, en edades comprendidas entre los 13 a 15 años de edad, los mismos constituyeron una sección. Según sus edades, se supone que estos jóvenes mantienen una estructura de pensamiento formal, siendo capaces de pensar en términos abstractos, de formular hipótesis e ir más allá del asunto estudiado a objeto de establecer sus causas y efectos como un medio para la resolución de problemas. El informante clave fue el docente del aula, quien participó de forma voluntaria.

\section{Técnicas de Investigación e Instrumentos de Recolección de Datos}

Las técnicas utilizadas para acceder a la información fueron la observación participante y la entrevista. Mediante la observación se registraron los sucesos, describiéndose los escenarios, las actividades y a los actores del aula, así como los detalles de los diálogos, las condiciones en que ocurrieron los fenómenos y las conductas de los estudiantes en los equipos cooperativos. 
Los registros descriptivos permitieron precisar cómo el aprendizaje cooperativo logra en los estudiantes el desarrollo de sus habilidades cognitivas. El uso de la entrevista tuvo como objeto recabar información acerca de lo aprendido durante el desarrollo de la experiencia, constatar situaciones observadas y precisar la manifestación de actitudes y emociones, entre otras cosas. El instrumento utilizado para la recolección de los datos fue el Diario de Notas y Anécdotas.

\section{Descripción de los Métodos de Aprendizaje Cooperativo}

Se aplicaron dos técnicas de aprendizaje cooperativo: "Aprendiendo Juntos" y "El rompecabezas", los dos descritos por Slavin (1999).

“Aprendiendo Juntos". Se conforman los equipos de manera espontánea, cuidando que en cada uno de ellos, participen estudiantes de ambos sexos y de distintos promedios en sus calificaciones. A cada uno de los equipos les fue entregado el material disponible para el desarrollo de la investigación: libros de diferentes autores, láminas de obras de arte, fotografías, entre otros materiales. Posteriormente, el docente realiza una descripción del tema objeto de estudio y asigna a cada equipo la responsabilidad de un contenido específico. En el equipo, sus miembros se distribuyen responsabilidades, cada uno con una tarea en particular a fin de profundizar, discutir, analizar y explicar el contenido. Elaboran un informe sobre el tema y luego lo exponen a los otros equipos. Para la evaluación se consideran la exposición de un alumno por cada equipo, el informe y una prueba escrita individual. Las calificaciones se obtienen por el promedio de notas logradas internamente en cada equipo.

"El rompecabezas". Se conforman grupos heterogéneos, en cuanto sexo, actitud y rendimiento, de 3 a 6 estudiantes, designándose uno de ellos como coordinador. El material a trabajar es dividido entre el número de participantes en cada equipo, indicándose el tiempo para su lectura y desarrollo. Luego los estudiantes con el mismo segmento de contenido se reúnen y conforman el "grupo de expertos temporales". Discuten y comprenden los contenidos del material; preparan y ensayan las presentaciones que harán a su equipo inicial. Al culminar esta discusión regresan a sus equipos originales, y socializan el resultado de la discusión. Cada estudiante presenta su segmento a su grupo. El profesor pasa de grupo en grupo para mediar cualquier situación que se presente y observar el proceso. Cuando cada uno de los miembros del equipo culmina la presentación que le corresponde, arman el rompecabezas del material que les fue asignado presentando un solo informe por equipo. El docente elige un miembro de 
cada equipo para la presentación final, y luego todos los estudiantes son evaluados en forma individual.

\section{Procedimiento}

El primer paso fue negociar con las autoridades de la institución a objeto de lograr su aprobación para la aplicación del aprendizaje cooperativo en el aula. Se decidió aplicar los métodos en el octavo grado y con la asignatura de Educación Artística. Posteriormente, se sostuvieron reuniones con la profesora de la cátedra, registrándose las primeras notas acerca de la situación inicial del estudiantado y el modo en que la docente llevaba a cabo las clases, para luego visitar el aula destinado a esa cátedra y realizar las observaciones pertinentes. Después se llevaron a cabo diversas reuniones de planificación. Durante las reuniones la docente se preparó para aplicar las técnicas, leyendo los procedimientos según la bibliografía consultada. Se conversó con el alumnado acerca de la aplicación de los métodos de aprendizajes, explicándole los objetivos y procedimientos a seguir.

La investigadora se incorporó a las clases de la asignatura de Educación Artística para registrar los eventos ocurridos en los equipos durante la aplicación de los métodos. Se describieron las actitudes, emociones, y todo aquello que respondían al desarrollo cognitivo y a las categorías inherentes al objeto de estudio. Al finalizar la aplicación se entrevistaron a los alumnos y a la profesora de la cátedra. Las entrevistas se refirieron acerca de los aprendizajes adquiridos, la manera en que los estudiantes experimentaron las aplicaciones, los alcances de trabajar en equipo, entre otros aspectos.

Se transcribieron las entrevistas y las observaciones realizadas, describiéndose los procesos de aplicación del aprendizaje cooperativo y construyéndose las categorías mediante el procedimiento descrito por Pérez (1994). A fin de realizar generalizaciones se detectaron en las descripciones los fenómenos que contenían elementos para explicar e interpretar, ya sean expresiones o actitudes que indicarán el desarrollo de las habilidades cognitivas. Las categorías fueron creadas con los fragmentos de las entrevistas realizadas a los alumnos y a la docente. En esta medida se realizaron las interpretaciones considerando las relaciones entre diversas categorías y eventos observados. Por último, se construyeron las conclusiones y se plantearon las recomendaciones. 


\section{Análisis de Datos}

El proceso seguido para el análisis de los datos consistió en tres momentos: descripción, análisis e interpretación de la información y de las situaciones (Pérez, 1994). La descripción y el análisis fueron sistemas que se dieron en conjunto. Tanto el análisis inicial como el estudio del contexto estuvieron basados en los datos tomados de los registros, notas de campos, entrevistas y observaciones. En la medida que se desarrollaba el análisis surgieron las categorías, esto permitió clasificar las descripciones en una secuencia lógica que ayudara a visualizar el proceso de aplicación del aprendizaje cooperativo. La descripción de las categorías condujo a establecer patrones en los datos y estos fueron reducidos mediante un examen de segmentos de textos. Otro sistema utilizado consistió en la utilización de segmentos como unidades de análisis para definirlas mediante categorías, colocando los fragmentos extraídos al lado de las cualidades creadas producto del proceso del análisis y teorización.

La interpretación consistió en integrar, relacionar y establecer conexiones entre las diferentes categorías creadas, haciendo comparaciones lógicas entre ellas, permitiéndose la elaboración de nuevas relaciones y facilitándose la oportunidad para volver sobre los datos tantas veces fuese necesario a fin de establecer consideraciones teóricas de gran validez sobre el asunto estudiado. La información se legitimó por negociación (Pérez, 1994; Martínez, 1998). Este proceso se llevó a cabo durante todo el tiempo de intervención en el aula, ya que la investigadora, luego de hacer las observaciones, compartía sus apreciaciones e interpretaciones con la docente que aplicaba el aprendizaje cooperativo. Este modo de legitimación permitió confiar en la información obtenida. En diversas oportunidades se preguntaron a los estudiantes acerca de situaciones observadas, como una manera de precisar la información y aceptarla como válida.

\section{RESULTADOS E INTERPRETACIONES}

Fueron desarrolladas nueve categorías de análisis (CA) a partir de la saturación de las evidencias descubiertas ya sean mediante las observaciones o las entrevistas, estas categorías son las siguientes:

CA1. Las interacciones dentro de los equipos; CA2. El sistema de adquisición de la información; CA3. La demostración de habilidades de procesamiento; CA4. La demostración de habilidades de transferencia; CA5. La flexibilización de los modelos mentales; CA6. El 
desarrollo del hábito de la disciplina; CA7. El seguimiento de instrucciones y la responsabilidad en aprender; CA8. El logro de la comprensión de los materiales trabajados; CA9. El aporte de los conflictos socio - cognitivos.

CA1. Las interacciones dentro de los equipos. A través de las interacciones los alumnos desarrollan sus expresiones verbales: "Se expresan oralmente de manera coherente, clara y precisa", - dice la docente, y sienten la necesidad de aprender, y comprender que muchas respuestas las tienen sus compañeros de equipo: "Formulaban preguntas para aclarar dudas. Formulan y reformulan sus planteamientos o puntos de vista". - aclara la profesora. Los estudiantes reconocen que las habilidades se logran mediante las relaciones de ayuda mutua: "Es bueno trabajar en equipo, ya que nos podemos ayudar entre nosotros mismos", de manera que los procesos de transferencias terminan siendo seguros: "Ahora cometo menos errores en los exámenes individuales y en las defensas del grupo".

Para algunos de ellos, la discusión interna de la temática en el equipo les permitió la adquisición del aprendizaje: "Discutimos y así puedo entender mejor lo que nos preguntan", y sus habilidades podían ser demostrables mediante las actividades comprensivas: "Mis compañeros me ayudan a comprender lo que vamos leyendo, lo explicamos y hasta buscamos cualquier ejemplo que nos ayude a mejorar, yo no entendía algunas cosas y me la explicaron, ahora te las puedo explicar a ti."- señalaba un alumno.

Uno de los principios del aprendizaje cooperativo es la ayuda mutua, su importancia radica en que facilita la transmisión de la información, y los miembros de los equipos, en su mayoría, aprenden a estar pendientes de que cada integrante de su grupo domine el material estudiado y comprenda el tema tratado, esto viene dado por el grado de cohesión grupal que depende de la "disposición para la solidaridad, la ayuda mutua, el respeto a las diferencias y la convivencia" (Pujolas y Lagos, 2011, p.44). La ayuda mutua facilita la confianza entre los integrantes de un equipo: "Con este tipo de actividad he aprendido a confiar en los demás, antes no confiaba", "El apoyo ayuda", son algunas apreciaciones de los estudiantes al experimentar el aprendizaje cooperativo.

CA2. Sistema de adquisición de la información. Una estudiante señalaba lo siguiente:

Es agradable, se me hace sencillo el aprender así, si no entiendo algo mi compañero sin molestarse me lo aclara, si yo lo entiendo antes se lo aclaro a ellos, comparto y cuando no recordamos algo, alguno lo recuerda y así todo el trabajo sale, no como cuando uno está solo con el libro, pienso que si alguno no está preparado actuando entre todos, se construye 
mejor la información, es productivo trabajar en equipo y debería ser en todas las asignaturas.

Contar con el compañero y entenderlo a él con mayor facilidad que al docente, tiene que ver con los cambios de roles y de liderazgo que se dan dentro de los equipos de cooperación. La cercanía entre los jóvenes permite que la adquisición se haga mediante la discusión y los aportes de cada uno de los integrantes del equipo. Siempre para los alumnos es necesario e importante entender, aunque muchas veces esta comprensión viene dada a través de sistemas individualizados donde la lectura y el estudio a solas ha sido la forma más usual para la adquisición. El aprendizaje cooperativo rompe con este esquema de adquisición, desde su aplicación se considera que la discusión es una herramienta cargada de elementos y contenidos que hacen que la persona aprenda: "Cuando discuto con mis compañeros el tema asignado, cada uno aporta y creamos conceptos más amplios y completos, vemos el tema explicado por el otro compañero y es más fácil de entender" - explicaba un estudiante, al consultársele sobre el tema.

Por otra parte, para muchos estudiantes las dificultades con las lecturas se debió al hecho de que las mismas no eran motivadoras. El leer facilita la adquisición de habilidades cognitivas, su énfasis está muy relacionado a la exclusividad de la adquisición del conocimiento y de la información, y es necesario como herramienta para el desarrollo humano y espiritual de la persona. Muchos son los estudiantes que huyen de la lectura, prefieren no hacerla, ya sea porque no saben, porque no comprenden o por el simple hecho de que no gustan leer. La lectura es una herramienta esencial del aprendizaje cooperativo, se realiza de un modo compartido acercando al alumno a la comprensión de los contenidos teóricos y a compartir las diferentes visiones de un mismo tema.

El hecho que los estudiantes realicen lecturas de manera compartida y socializada, es significativo para ellos, ya que de esta manera se hacen responsables de brindar a sus compañeros su visión y explicación del asunto (Slavin 1999), de este modo el proceso de adquisición es diferente, así como los procesos de flexibilización de los modelos mentales. Los espacios de lectura que puedan darse en los equipos cooperativos, facilitan el desarrollo de competencias orales y permiten situaciones comunicativas en las que el estudiantado aprende a reflexionar acerca del uso de la lengua. Estos intercambios ayudan a razonar y a experimentar estrategias comunicativas y lingüísticas que facilitan el desarrollo de la escucha activa y solidaria (Pujolas, 2002) 
CA3. Demostración de las Habilidades de Procesamiento. En la medida que los estudiantes discutían y parafraseaban el contenido que leían se revelaban sus habilidades de procesamiento de la información, así como también cuando, en los equipos, se preguntaban entre sí acerca de los contenidos. Todos los miembros de los equipos fueron capaces de formar redes de conocimientos, al hacer inferencia y al presentar sus materiales ya sea en una exposición, collage, mapa mental o conceptual. Pero en este caso, la discusión actuó como canalizador de las redes originadas en cada uno de los miembros de los equipos. Si no discutían no podían reconocer que aprendían, mucho menos saber la calidad de los aprendizajes obtenidos.

Es más cómodo, cuando discutimos el tema, las pinturas, el tipo de arte, de dónde es la expresión, todos opinamos, investigamos en nuestros materiales, discutimos y decidimos, o sea comprendemos mejor, se hace más corto el tema y las respuestas son más precisas.

El debatir las ideas supone una manera de afianzar el contenido y el conocimiento del mismo, la socialización facilitó la sistematización de una buena parte del conocimiento, la cual permitió la teorización y las propuestas (Aranguren, 2014).

CA4. Demostración de Habilidades de Transferencia. La naturaleza del aprendizaje cooperativo facilitó la transferencia de la información, dado su carácter promotor de discusiones y debates: "Hemos aprendido a escuchar, leemos y aportamos ideas a veces unas muy diferentes de otras...". Durante el desarrollo del trabajo en equipo los estudiantes leen y ofrecen sus ideas: "Puedo dialogar, es más eficaz discutir un tema y sacar conclusiones con otro, es como la levadura crecen las conclusiones se hacen más consistentes. - dijo un estudiante al ser consultado.

Las habilidades de transferencia se desarrollan con la participación. El aporte de ideas, el diálogo o la conversación, son los mecanismos de mayor poder que se descubre en el aprendizaje cooperativo para transferir lo que se aprende en la dinámica dada en los equipos. Cuando los jóvenes discuten y explican los contenidos adquiridos, flexibilizan sus pensamientos y pueden cambiar de idea con mayor facilidad.

En el desarrollo de las habilidades de transferencias se hace importante la aparición del "otro significativo", Ovejero (1990) lo define como el estudiante, de modo especial el compañero de equipo, que espera y recibe conductas y actitudes positivas de sus demás pares y que fortalecen, significativamente, a nivel interpersonal e intelectual al equipo. Lo importante 
es hacer que los otros significativos se conviertan en actores que aporten y fortalezcan las habilidades cognitivas. Para esto es imprescindible la atención del docente en las formas y maneras que se resuelven las relaciones internamente en el grupo general, a fin de ayudar a los alumnos a escoger quién o quiénes serían sus compañeros de equipos.

Cuando un alumno descubre que su compañero es un amigo significativo para él, la transferencia y la forma de sistematizar sus aprendizajes y conocimientos se transforma, sin perder la esencia del lenguaje común (Aranguren, 2003):

La aplicación del aprendizaje cooperativo es un modelo para ayudar al estudiantado a sistematizar sus experiencias y conocimientos, sólo se da en la medida que el estudiante, en conjunto con sus otros significativos, clasifique mentalmente lo aprendido y aplique, correctamente, todos los conocimientos y aprendizajes en nuevos contextos y situaciones dadas en el aula de clases.

CA5. La flexibilización de los Modelos Mentales. Una manera de flexibilizar los modelos mentales durante el ejercicio de trabajo en equipo es la escucha y la participación oportuna: “(...) doy mi opinión y escucho, así nos reforzamos en los conceptos...”- afirmaba una estudiante al ser consultada. Las discusiones y los debates son estrategias internas del aula que facilitan la flexibilización de las formas de pensar. En estos procesos es imprescindible la valoración de las ideas y el reconocimiento de las capacidades cognitivas de los pares, eso ayuda durante el sistema de adquisición:

Me gusta porque me valoran y valoro las ideas de los otros, ya no me quedo solo con mi opinión; además, en esta clase hay gente que conoce de los temas más de lo que uno piensa y tienen unas historias muy buenas que ayudan a uno a comprender y a ubicarse en la idea que uno tiene.

En dicha reflexión se encuentra implícita la capacidad que tiene la persona de flexibilizar sus modelos mentales. Esta flexibilización pasa por un proceso de cambio de paradigmas y de ideas: "A veces cuando llegamos con una idea nos vamos con otra" - señalaba una estudiante, y de ese modo se fortalecen los conocimientos y los aprendizajes adquiridos:

A mí, personalmente, me ha agradado porque he aprendido a intercambiar mis ideas, ya no me quedo con lo que sé, ahora disfruto cuando lo comparto y sé que ellos también disfrutan compartiendo lo que saben, ahora aprendo mucho más rápido, le comprendo más cuando mis compañeros me explican.

Aprender a intercambiar las ideas es un ejercicio mental que implica reflexión y concienciación acerca de lo que se piensa sobre algún aspecto en particular, demuestra una 
capacidad de reconocimiento de las limitaciones, es una genuina forma de demostración de la metacognición como el grado de conciencia que tiene la persona acerca de su forma de pensar, (Pérez, Escolano, Pascual, Lucas y Sastre, 2015). El hecho que a un estudiante no se le olvide el material aprendido tiene que ver con el modo en que adquiere la información, la integra y la interrelaciona con todos los conocimientos previamente logrados y con la aplicación debida de los aprendizajes.

CA6. El desarrollo del hábito de la disciplina. En la medida que los estudiantes realizan sus trabajos y estudios mediante el aprendizaje cooperativo desarrollan la disciplina como hábito, esto contribuye a la comprensión de los contenidos y facilita la seguridad en los miembros de los equipos: "Me siento bien si todo el equipo lo toma en serio y trabajan", afirmaba un estudiante. Los estudiantes reconocen que un trabajo disciplinado redunda en trabajos de calidad: "Cuando te das cuenta que el trabajo en equipo te ayuda a comprender mejor los contenidos y por ende vas a salir mejor en las evaluaciones entonces se ponen serios y trabajan, es cuestión de tiempo y conciencia”. La disciplina, entendida como la capacidad y voluntad interna de responder de forma planificada, responsable y consciente a las obligaciones y compromisos, conduce al aumento de la capacidad comprensiva y al desarrollo de las habilidades intelectuales en cada miembro de los equipos, todo esto hace que los estudiantes reconozcan la necesidad de conformar equipos con personas de alto nivel de responsabilidad: “Tienes que contar con personas que tomen en serio la actividad.”, decía una estudiante en cuanto a esto.

Por otra parte, el seguimiento de las instrucciones y el logro de los acuerdos entre los estudiantes contribuye a disminuir las tensiones internas en los equipos, mejorándose las interacciones y la convivencia (Herrada y Baños, 2018). Si las normas a seguir durante la actividad están claras y responden a un proceso previo de negociación hace que todos sientan responsabilidad, influyendo esto en la disciplina del aula. Los acuerdos logrados entre los estudiantes facilita el control interno de los grupos. Permitir que los estudiantes propongan sus normas, ayuda a que las mismas se cumplan y se mantengan en el tiempo, redundando en la formación de los hábitos sociales y mentales. El control de la disciplina y el seguimiento de las instrucciones son mecanismos importantes para desarrollar habilidades cognitivas, ya que es imposible que los estudiantes desarrollen sus habilidades en ambientes cargados de molestias y 
rabias, así como de ruidos y de distractores como las interrupciones reiteradas por diversos agentes externos.

CA7. El seguimiento de instrucciones y la responsabilidad en aprender. El énfasis del seguimiento de las instrucciones y el control de la disciplina en el aula ayuda al estudiante a permanecer en un estado mental disponible para el aprendizaje, facilitándole demostrar con soltura las distintas formas de cómo alcanzó comprender los contenidos.

Por otra parte, el reiterado mecanismo de hacer que los alumnos sean responsables con sus equipos permite que todos estén dispuestos a transferir los conocimientos:

Cuando la responsabilidad es compartida, todos tenemos que estar pendiente de que cada uno aprenda para poder defender al grupo, eso nos hace más críticos, más solidarios y persistentes en conseguir ser mejores, por eso leemos, volvemos a leer, discutimos y llegamos a conclusiones, a veces diferentes, entonces volvemos a leer, hasta entender.- dice un alumno.

Los estudiantes pueden tender a creer que hay margen para dejar que el otro haga toda la actividad, pero cuando se aplica el aprendizaje cooperativo y los alumnos descubren que este sistema tiene reglas que son necesarias cumplirlas para alcanzar el nivel del equipo, entonces comienzan a verse los cambios en ellos: "Yo soy el responsable del trabajo que nos mandan y cada uno de nosotros es responsable" - señalaba un estudiante. La responsabilidad de que el otro adquiera el conocimiento que "yo poseo" se hace responsabilidad de cada uno de los miembros de los equipos: "Antes de cerrar cada uno pregunta lo que no ha aprendido y el que más lo domina se lo explica y así hasta terminar. Se hace evidente cuando se demuestran los éxitos, y cuando el docente los afianza con expresiones motivadoras y positivas, porque fortalece los procesos en el futuro. En este sentido, la aplicación de esta metodología disminuye la dependencia de los estudiantes de sus profesores, y aumenta la responsabilidad de los educandos por su propio aprendizaje y el de sus compañeros de equipo: "Me siento incomodo si no leo antes de venir a clases porque sé que los demás han leído. Traigo material y mis preguntas...", "Al final de la actividad todos debemos dominar el tema", "Somos responsables de que lo que nos tocó este bien investigado, con las respuestas correctas, porque de eso depende el trabajo de todos. - son básicamente las apreciaciones de los estudiantes en cuanto su responsabilidad.

El trabajo cooperativo brindó la posibilidad de que los estudiantes trabajasen seguros, en confianza y animados por sus compañeros de equipo. Haciéndolos más responsables en 
adquirir nuevos aprendizajes y conocimientos en equipo. En este sentido, la ayuda mutua se convirtió en una clave importante en el desarrollo de la comprensión y de las habilidades cognitivas, ya que contribuye en generar confianza en los estudiantes: "Pero confiamos en el trabajo que el otro hace y al final nos consultamos antes de hacer el informe final”, "A mí me da más confianza, y salgo adelante con el trabajo”, "Cuando trabajo en equipo me siento más segura”, - afirmaban los estudiantes al ser consultados.

CA8. El logro de la comprensión de los materiales trabajados. Una de las ventajas de la aplicación del aprendizaje cooperativo es su contribución al desarrollo de la comprensión, eso lo afirmaron los estudiantes al ser entrevistados: "Yo comprendo mucho más cuando estoy trabajando con mis compañeros en equipos pequeños que cuando lo hago solo", "Si trabajo en equipo y hay algo que no sé o no entiendo, uno de ellos me lo explica y logro comprender”, "En el equipo discutimos, analizamos, buscamos entre todos ejemplos dando cada uno su aporte", "Cuando trabajamos en equipo, todo se me hace más sencillo, compartimos las ideas, intercambiamos opiniones y concluimos". En este sentido, es importante destacar que esta metodología favorece la interconexión y la aplicación de los conocimientos con las experiencias de la cotidianidad, (Madrid, Arellano, Jara,Merino y Balocchi, 2015) siendo esto un modo de desarrollo de la comprensión.

La comprensión es una práctica que implica el fortalecimiento de muchas actividades y estrategias que colocan a la persona a pensar de manera diferente al modo que ha venido haciéndose. Esto es difícil en la escuela, debido a que los modos de comprensión de los asuntos siguen siendo los mismos de siempre, es fácil observar que lo más importante es que el estudiante memorice tablas, fórmulas, procedimientos; pero la interpretación adecuada de la simbología, el entendimiento del problema como un sistema de la vida cotidiana y la capacidad de ver las situaciones históricas en un contexto donde intervienen muchos aspectos exige un mayor nivel de comprensión y una astucia por parte del docente para llevar al estudiante a lograr un nivel de análisis mucho mayor. Muchos estudiantes demostraron problemas concretos en cuanto a la comprensión de los materiales, descubriéndose dificultades en cuanto a los modos de explicar o justificar situaciones, y todo porque no poseen estructuras mentales que sólo se desarrollan con la formación de los hábitos y con la práctica permanente de estrategias cognoscitivas, tanto de procesamiento como de ejecución (Chadwick, 1988). 
CA9. El aporte de los conflictos socio-cognitivos. Durante todo el proceso de aplicación del aprendizaje cooperativo los estudiantes experimentaron la discusión y el diálogo dentro de los equipos. Ello constituyó la pieza fundamental de los análisis e interpretaciones de los contenidos: "muchas mentes piensan mejor que una, todos tenemos distintos puntos de vista, aportamos y además es bueno saber lo que piensa cada uno del equipo para luego construir una sola idea, trabajando en grupo uno termina con mayor conocimiento sobre el tema, podemos discutir más, y si uno tiene alguna complicación, otro compañero lo explica... "esta apreciación señalada por una estudiante, refleja el sistema de discusión interna en los equipos, elemento imprescindible en la revelación de los conflictos socio - cognitivos. El logro del aprendizaje como consecuencia de estos conflictos se basa en la confrontación de los diferentes puntos de vista: "Yo creo que trabajando en equipo podemos comprender mejor lo que estas estudiando o trabajando, porque todos tienen diferentes puntos de vista y todos al final tenemos la razón...", esto genera el desequilibrio cognitivo y ayuda, a los que se encuentran en ese conflicto, a construir una estructura mental más compleja.

\section{CONCLUSIONES}

Con base en las interpretaciones anteriores, se señalan las siguientes conclusiones:

-El aprendizaje cooperativo facilita que los estudiantes manifiesten lo que aprenden con mayor soltura mediante el desarrollo de actividades comprensivas; por tanto, el logro de estas habilidades se alcanza mediante la interacción entre los miembros de los equipos, ya que en la medida que los estudiantes socializan adquieren, elaboran y transfieren el conocimiento y sus aprendizajes.

-Las habilidades de adquisición se logran mediante el ejercicio de la lectura, siendo la conversación un mecanismo para afianzar el aprendizaje. La confrontación de puntos de vista diferentes y la explicación por parte de un actor más avanzado, permite que los estudiantes reorganicen sus estructuras mentales y crean nuevos modelos, constatando con esto, la propuesta teórica de Vygotsky (1979) referente a la zona de desarrollo próximo.

-Para la aplicación del aprendizaje cooperativo es importante considerar el número de integrantes del equipo, el cual debe variar según el tiempo que se le destine al trabajo a realizar. Cuanto menos tiempo haya para trabajar, menos integrantes debe tener un equipo, la idea es que el tiempo de discusión sea equitativo con el número de integrantes. de manera que 
las exigencias en cuanto al producto, estén en concordancia con estas condiciones. La heterogeneidad de los equipos aumenta la posibilidad de puntos de vista diferentes, a su vez permite que los estudiantes menos aventajados puedan verse favorecidos y acompañados.

-El aprendizaje cooperativo facilita la creación de modelos mentales flexibles. La discusión hace que los estudiantes descubran que a veces no saben con certeza algún contenido, o poseen una falsa o inadecuada conceptualización de los asuntos, generando un cambio en sus ideas. Para ello, son necesarios los conflictos socio - cognitivos que afecta positivamente el trabajo en equipo, facilitando la elaboración del conocimiento y haciendo al estudiante más habilidoso en su transferencia.

-Experimentar los métodos de aprendizaje cooperativo les permitió a los estudiantes una nueva manera de aprender. Con su aplicación el alumnado trabaja tres niveles de comprensión: el de contenido, resolución de problemas y el de investigación (Perkins, 1999). El de contenido lo alcanza mediante la adquisición del conocimiento a través de la lectura, el parafraseo y repetición de los temas durante las discusiones; la resolución de problemas se observa en los modos en que resuelven los asuntos que les ocasiona interferencia para adquirir, procesar y transferir la información, y el de investigación lo logra mediante el proceso de indagación y sistematización de la información.

-Durante la aplicación del aprendizaje cooperativo se descubren una serie de elementos y circunstancias que afectan el desarrollo de las habilidades cognitivas. Las mismas dependen de las situaciones internas de la persona, las relaciones entre los alumnos, el ambiente, los valores y las estratégicas en los procesos de enseñanza y aprendizaje. De manera positiva se revela el desarrollo de la confianza, la interdependencia positiva, la flexibilización de los modelos mentales, la comprensión del tema, la ayuda mutua, el compartir los materiales de estudio, las discusiones entre los alumnos, la valoración de las ideas, la interacción y la participación en los equipos. De manera negativa, se descubre el miedo a exponer, la inseguridad, las estructuras mentales muy rígidas, la actitud "cerrada" y autoritaria del docente, no encontrar a la persona indicada para la ayuda, la excesiva dependencia a lo que señale el profesor, el uso de materiales muy extensos, poco tiempo para el estudio y análisis de los materiales, las actividades académicas sin sentido significativo para los alumnos, la indisciplina, las condiciones mínimas para el desarrollo de las actividades académicas, así como la poca o nula oportunidad para compartir aprendizajes. 
- Finalmente, el dominio de las habilidades depende de la orientación de las acciones que se ejecutan en el proceso de enseñanza y de aprendizaje. Para que un estudiante domine sus habilidades se requiere un sistema que lo consolide mediante un ejercicio permanente. Las acciones para el dominio de las habilidades cognitivas deben hacerse de forma frecuente y periódica, por ello el uso de algunas estrategias convencionales no facilitan el hábito por ser esporádico el tratamiento en el proceso. En cambio, el uso reiterado y frecuente de técnicas de aprendizaje cooperativo afianza las condiciones para que el estudiante adquiera, procese y transfiera sus aprendizajes.

\section{REFERENCIAS}

Aranguren P. G. (2003). La función etnográfica de la hermenéutica, la sistematización y la ética, en la comprensión de los textos representativos de los actos de vida. Revista Educación y Ciencias Humanas. (20) 15 - 40.

Aranguren, P. G. (2014). El debate cooperativo versus la exposición verbal del docente. Revista Educare [Revista en Línea], 18 (1) 26 - 49. Disponible: http://revistas.upel.edu.ve/index.php/educare/article/view/1868

Azorín, A. C. M. (2018). El método de aprendizaje cooperativo y su aplicación en las aulas. Perfiles Educativos [Revista en Línea], 40 (161) 181 - 194. Disponible: https://www.iisue.unam.mx/perfiles/numeros/2018/161

Candela, A. (1995). Transformaciones del conocimiento científico en el aula. En: Rockwell, E. (Coord.) La Escuela Cotidiana. (pp. 173 - 197). México: Fondo de Cultura Económica.

Capilla, R. M. (2016). Habilidades cognitivas y aprendizaje significativo de la adición y sustracción de fracciones comunes. Cuadernos de Investigación Educativa [Revista en Línea], 7 (2) 49 - 62. Disponible: http://www.scielo.edu.uy/pdf/cie/v7n2/v7n2a04.pdf.

Chadwick, C. B. (1988). Estrategias cognitivas y afectivas de aprendizaje. Revista Latinoamericana de Psicología. 20 (2). 162 - 205.

Chadwick, C. B. y Rivera, N. I. (1991). Evaluación formativa para el docente. Barcelona: Paidós.

De Sánchez, M. (1991). Desarrollo de habilidades del pensamiento: procesos básicos del pensamiento. México: Trillas.

Flores, L. O. (2015). La participación de los estudiantes en el aula como factor determinante para mejorar la calidad de los aprendizajes. Estudio sobre la importancia que le otorgan los profesores estudiantes de Enseñanza Media del Colegio San Ignacio Alonso Ovalle a la participación en el aula. Trabajo de grado de Maestría no publicado. 
Universidad Alberto Hurtado, Facultad de Educación. Disponible: https://doi.org/10.25100/rc.v20i2.4609

Gagné, R. M. (1987). Las condiciones del aprendizaje. (4ta. edic.). México: McGraw - Hill.

García, V. M. C. (2018). Competencias claves y aprendizaje cooperativo, conceptos fundamentales en la educación actual. International Studies on Law and Education [Revista en Línea], (29/30). 79 - $90 . \quad$ Disponible: http://rua.ua.es/dspace/handle/10045/72368

Gilar, R. C. (2003). Adquisición de habilidades cognoscitivas. Factores en el desarrollo inicial de la competencia experta. Trabajo Doctoral no publicado. Universidad de Alicante. Disponible:

http://descargas.cervantesvirtual.com/servlet/SirveObras/0137270861135951475680 2/011762_1.pdf

Johnson, D y Johnson, R. (1999). El Aprendizaje cooperativo en el aula. Buenos Aires: Paidós.

Hernández, Y. C. y Aranguren, P. G. (2016). Patrón tecnopedagógico: ruta de aprendizaje basado en actividades comprensivas. Revista Vínculos [Revista en Línea], 13 (2) 30 39.. Disponible: http://oaji.net/articles/2017/3374-1493745512.pdf

Herrada, V. R. I. y Baños, N. R. (2018). Revisión de experiencias de aprendizaje cooperativo en ciencias experimentales. Campo Abierto [Revista en Línea], 36 (2) 157 - 170. Disponible: https://mascvuex.unex.es/revistas/index.php/campoabierto/article/view/2987

Larraz, R. N. (2015). Desarrollo de las habilidades creativas y metacognitivas en la educación secundaria obligatoria. Madrid: Dykinson.

Loor, S. L.R; Palma, V. M. M; Saltos, R. L. J y Bolívar, Ch. O. E. (2018). El aprendizaje cooperativo como una estrategia de enseñanza del Idioma de Ingles en las Escuelas públicas del Ecuador. Revista Científica Dominio de las Ciencias [Revista en Línea], 4 (3) 431 - 448. Disponible: http://dominiodelasciencias.com/ojs/index.php/es/index

Madrid J. C.; Arellano, M.; Jara, R.; Merino, C. y Balocchi, E. (2013). El aprendizaje cooperativo en la comprensión del contenido "disoluciones". Un estudio piloto. Revista Didáctica de la Química [Revista en Línea], (24) 471 - 479. Disponible: https://www.sciencedirect.com/science/article/pii/S0187893X13725151

Martínez, M. (1998). La investigación cualitativa etnográfica en educación: Manual teórico práctico. (3era. edic.) México: Trillas.

Martinic, S. (2015). El tiempo y el aprendizaje escolar. La experiencia de la extensión de la jornada escolar en Chile. Revista Brasileira de Educação [Revista en Línea], 20 (61) 479 - 499. Disponible: http://www.scielo.br/pdf/rbedu/v20n61/1413-2478-rbedu-20-610479.pdf 
Morales, M. L.; García, M. O.; Torres, R. A. y Lebrija, T. A. (2018). Habilidades Cognitivas a través de la Estrategia de Aprendizaje Cooperativo y Perfeccionamiento Epistemológico en Matemáticas de Estudiantes de Primer Año de Universidad. Formación Universitaria [Revista en Línea], 11 (2) 45 - 56. Disponible: http://dx.doi.org/10.4067/S0718$\underline{50062018000200045}$

Mugny, G. (1988). Psicología Social de Desarrollo Cognitivo. Madrid: Antrópodos.

Muñoz, G. M. (2017). Implicaciones del desarrollo de habilidades del pensamiento en el desempeño académico de los alumnos de preparatoria. En: Hereida, E. Y. y Cannon D. B. Estudio sobre el desempeño académico. Ciudad de México: Nómada.

Ovejero, A. (1990). El aprendizaje cooperativo: Una alternativa a la enseñanza tradicional, Barcelona: Promociones y publicaciones universitarias.

Papalia, D. E; Wendkos, O. S. y Duskin, F. R. (2010). Desarrollo humano (11ava. edic.). México, D. F.: McGraw - Hill.

Pérez, I. A.; Escolano, P. E.; Pascual, S. M. T.; Lucas, M. B y Sastre, S. (2015). Metacognición en un proceso de aprendizaje autónomo y cooperativo en el aula universitaria. Contexto Educativo [Revista en Línea], (18) $95-108 . \quad$ Disponible: https://www.researchgate.net/publication/279158578_Metacognicion_en_un_proces o_de_aprendizaje_autonomo_y_cooperativo_en_el_aula_universitaria

Pérez, N. M. V. (2016). El desarrollo del pensamiento lógico verbal y abstracto en educación inicial. Trabajo de Grado de Maestría no publicado, Universidad Andina Simón Bolívar. Sede Ecuador. Área Educación Disponible: http://repositorio.uasb.edu.ec/bitstream/10644/5395/1/T2111-MINE-PerezEl\%20desarrollo.pdf

Pérez, S. G. (1994). Investigación Cualitativa. Retos e Interrogantes. (Tomo II). Madrid. La Muralla.

Perkins, D. (1999). La escuela inteligente. Barcelona: Gedisa.

Piaget, J. y Inhelder, B. (2015). Psicología del niño. (9na.edic.). Madrid: Morata.

Pujolàs, P. (2002). El aprendizaje cooperativo. Algunas propuestas para organizar de forma cooperativa el aprendizaje en el aula. [Documento en línea], Laboratorio de Psicopedagogía. Universidad de Vic, Zaragoza. Disponible: https://www.ugr.es/ fjirios/pce/media/7a-AprendizajeCooperativoAula.pdf

Pujolàs, P. y Lago, J. R. (coords.). (2011). El programa CA/AC ("Cooperar para aprender / aprender a cooperar") para enseñar a aprender en equipo. Implementación del aprendizaje cooperativo en el aula. [Documento en Línea], Barcelona: Universitat Central de Catalunya. Disponible: http://www.cife-eicaac.com/programa_ESP.asp 
Ramos, A.I.; Herrera, J. A. y Ramírez, M.S. (2010). Desarrollo de habilidades cognitivas con aprendizaje móvil: un estudio de casos. Comunicar, Revista Científica de Educomunicación [Revista en Línea], 17 (34) 201 - 209. Disponible: https://webcache.googleusercontent.com/search?q=cache:ALJ7YXnK9o8J:https:// www.revistacomunicar.com/verpdf.php\%3Fnumero\%3D34\% 26articulo\%3D34$\underline{2010-23+\& c d=16 \& h l=e s-419 \& c t=c \ln k \& g l=v e}$

Ríos, C. P. (2014). La aventura de aprender. (5ta. edic.). San Cristóbal: Cognitus.

Romero, C. Y. y Tapia, L. F. (2014). Desarrollo de las habilidades cognitivas en niños de edad escolar. MULTICIENCIAS [Revista en Línea], 14 (3) 297 - 303. Disponible: http://www.redalyc.org/pdf/904/90432809008.pdf.

Samaniego, C. M. (1995). La interacción entre compañeros: el conflicto sociocognitivo, el aprendizaje cooperativo y la tutoría entre iguales. Revista Interuniversitaria de formación del profesorado [Revista en Línea], (23) 177 - 186. Disponible: https://www.researchgate.net/publication/28254895_La_interaccion_entre_compan eros_el_conflicto_sociocognitivo_el_aprendizaje_cooperativo_y_la_tutoria_entre_ig uales

Slavin, R. (1987). Developmental and motivational perspectivas on cooperative learning: a reconciliation. Child Development. (58) 1161 - 1167.

Slavin, R. (1999). Aprendizaje Cooperativo. Teoría, investigación y práctica. Buenos Aires: AIQUE.

Vergel, O. M; Duarte, H. I. y Martínez, L. J. J. (2016). Desarrollo del pensamiento matemático en estudiantes de cálculo integral su relación con la planificación docente. Revista Científica [Revista en Línea], $23 \quad 17$ - 29. Disponible: http://revistas.udistrital.edu.co/ojs/index.php/revcie/article/view/8069/11025

Vygotsky, L. (1979). El desarrollo de los procesos Superiores. Barcelona: Critica. 